\title{
Enfermedad de Forestier-Rotes-Querol. Osificación del ligamento longitudinal cervical anterior como causa de disfagia
}

\author{
L. Alcázar; P. Jerez; J.C. Gómez-Angulo; M. Tamarit; R. Navarro; J.M. Ortega; P. Aragonés; F. Salazar y J.M. del Pozo
}

Servicio de Neurocirugía. Hospital Universitario de Getafe. Getafe. Madrid.

\section{Resumen}

La enfermedad de Forestier-Rotes-Querol o "hiperostosis esquelética idiopática difusa" es una enfermedad reumatológica con afectación sistémica ${ }^{18}$. Consiste en la calcificación-osificación del ligamento longitudinal común anterior (LLCA), de etiología aún desconocida, sin otros cambios degenerativos. La región más frecuentemente afectada en el raquis es la región dorsal baja ${ }^{18,28}$. La mayoría de casos se mantienen asintomáticos o con mínimos síntomas como dolor articular leve y dolor de espalda ${ }^{25}$. El síntoma más común cuando está implicada la columna cervical es la disfagia; menos frecuente es la disnea, ambos síntomas secundarios a la compresión extrínseca del esófago y la tráquea. La presencia de déficits neurológicos es rara ${ }^{18,21,25}$. En la década de los 70 se definieron criterios radiológicos específicos para el diagnóstico de la enfermedad de Forestier que todavía hoy se emplean ${ }^{18,28,29,30}$. Predomina en varones en la década de los 60 años ${ }^{18,25}$. Presentamos en este trabajo dos casos diagnosticados por alteraciones importantes en la deglución, una mujer de 84 años y un varón de 54 años de edad; ambos fueron intervenidos quirúrgicamente para la extirpación-fresado de una exostosis ósea de gran tamaño existente en C3-C4 y C5C6, respectivamente, mediante un abordaje anterior convencional a la columna cervical; la mejoría clínica fue inmediata tras la cirugía, con resolución completa del trastorno deglutorio.

PALABRAS CLAVE: Enfermedad de Forestier. Disfagia. Hiperostosis esquelética idiopática difusa. Osificación del ligamento longitudinal común anterior. Osteofito cervical.

Forestier-Rotes-Querol's disease. Ossification of the anterior cervical longitudinal ligament as a cause of dysphagia

\section{Summary}

Forestier's disease or diffuse idiophatic skeletal hyperostosis is a systemic reumathological abnormality of unknown etiology ${ }^{18}$. It produces calcificationossification of the anterior longitudinal ligament. The low dorsal region is the most affected in the raquis ${ }^{18,28}$. These patients are tipically asymptomatic or with few symptoms (minimal joint pain, spinal pain, stiffness) ${ }^{25}$. Dysphagia is the most common symptom when the disease affects the cervical spine; less frequent is dyspnea, both secondary to extrinsic compression of the esophagus and trachea. Neurological complaints are quite rare $^{18,21,25}$. In the 1970s Resnick described specific radiological criteria for the diagnosis of Forestier's disease that are still used today ${ }^{18,28,29,30}$. It affects men more frequently than women $(2: 1)$; the peak occurrence is in patients in their $60 \mathrm{~s}^{18,25}$. We present two cases diagnosed by severe difficulty with deglution, a 84 years-old woman and a 54 years-old man; we operated on them for surgical decompression of the esophagus with resection of osteophytes $\mathrm{C} 3-\mathrm{C} 4$ and $\mathrm{C5}-\mathrm{C} 6$ respectively through a conventional anterolateral neck approach. Relief of difficulty in swallowing was immediately ensued.

KEY WORDS: Forestier's disease. Dysphagia. Diffuse idiophatic skeletal hyperostosis. Ossification of the anterior longitudinal ligament. Cervical osteophyte.

\section{Introducción}

La enfermedad de Forestier, también conocida como "hiperostosis esquelética idiopática difusa" y ocasionalmente como "espondilosis hiperostótica" e "hiperostosis anquilosante" fue descrita por primera vez por Forestier y Rotes-Querol en $1950^{6,7,13,30}$; ellos eligieron el nombre de

Abreviaturas. LLCA: ligamento longitudinal común anterior. ORL: otorrinolaringólogo. RM: resonancia magnética. TC: tomografia computarizada. 


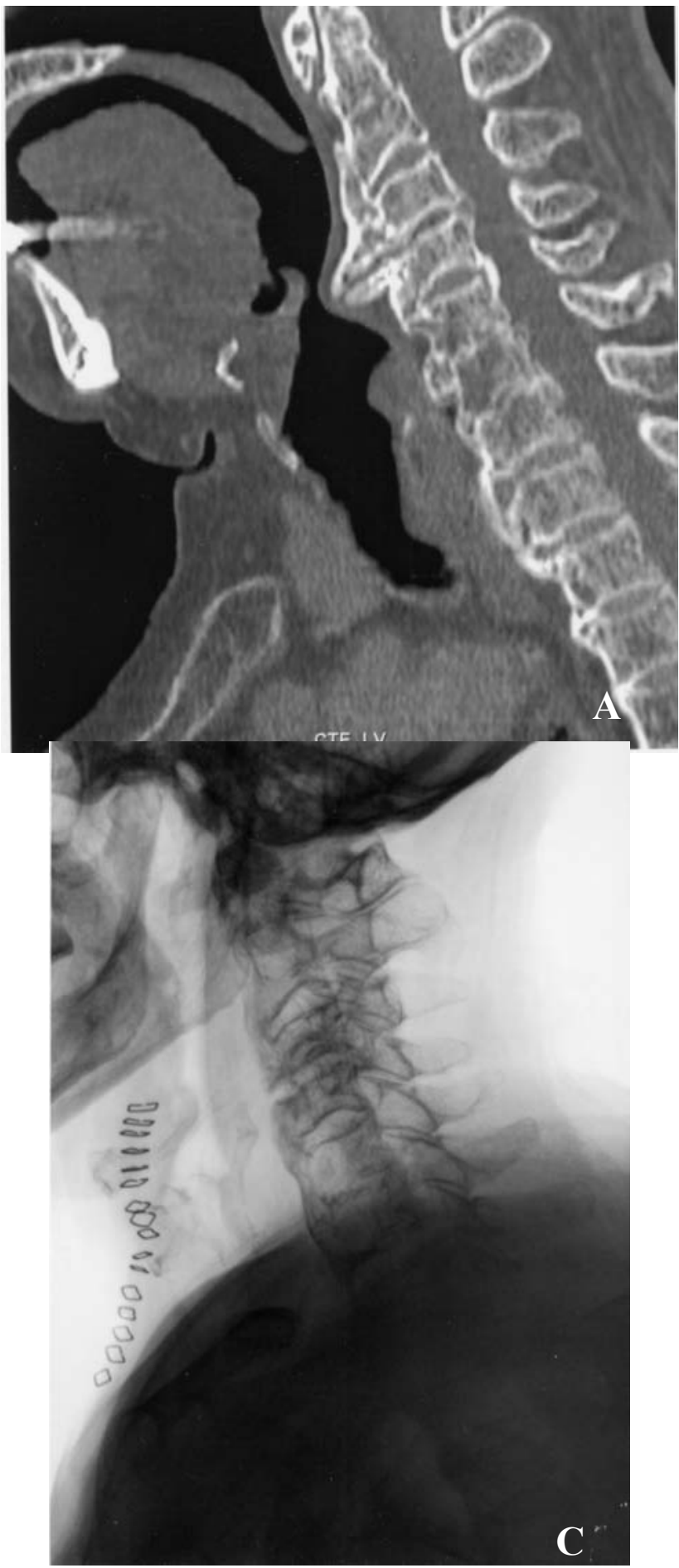

"hiperostosis anquilosante senil de la columna" para referirse a un conjunto de trastornos reumatológicos hallados en la población anciana, especialmente en varones de la sexta a la séptima décadas de la vida ${ }^{18,25}$; se definió como la osificación de la porción anterior y lateral derecha de los cuerpos vertebrales ${ }^{13}$. Más tarde, en 1971, Forestier y Lagier describieron la hiperostosis de la columna en adul-

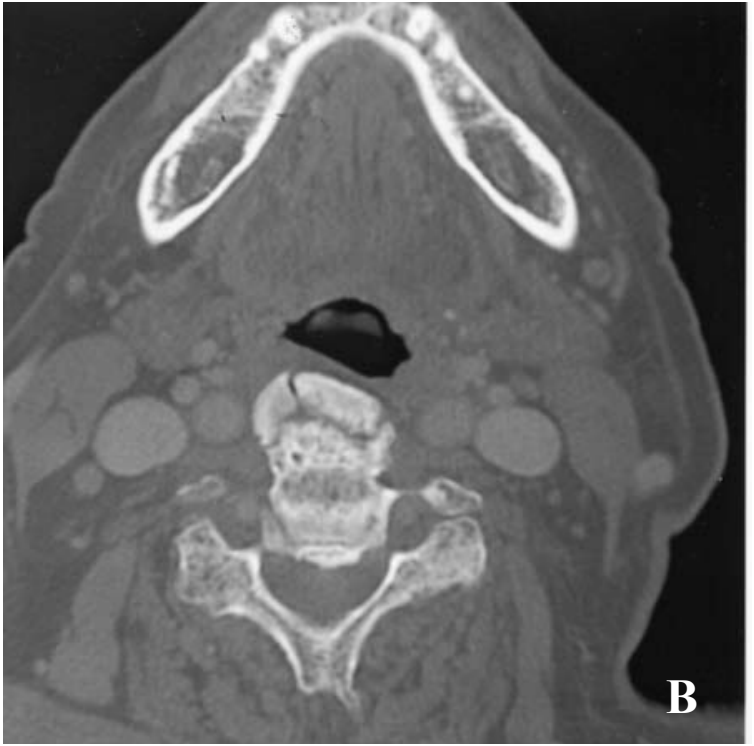

Figura 1 (Caso 1). A. Radiografía simple (proyección lateral) donde se observa la osificación del LLCA, muy llamativa en el interespacio C3-C4. B. TC (corte axial) que muestra la repercusión de la osificación del LLCA sobre la luz faríngea. C. Radiografía simple postoperatoria.

tos jóvenes y omitieron el término "senil". Anteriormente fue descrita en numerosas ocasiones y denominada de formas muy variables ${ }^{12,27,30}$, pero nunca categorizada como una entidad propia hasta 1950. La espondilitis anquilosante y la existencia de osteofitos vertebrales anteriores en el contexto de una enfermedad degenerativa de la columna cervical pueden ser confundidas con la enfermedad de Forestier; osteofitos radiológicamente significativos pero clínicamente asintomáticos u otras anomalías óseas hipertróficas están presentes en el $20-30 \%$ de la población ${ }^{15}$. Se trata de una enfermedad reumatológica sistémica que implica la existencia de una calcificación llamativa del LLCA y ausencia de otros cambios degenerativos ${ }^{18}$.

Los síntomas más comunes en la enfermedad de Forestier cervical son la disfagia y, menos frecuentemente, la disnea, secundarios éstos a una compresión mecánica del esófago y la tráquea ${ }^{5,8,10}$. La asociación de la enfermedad de Forestier y déficit neurológico significativo se ha publicado en raras ocasiones, debido éste a una estenosis del canal espinal cervical o torácico ${ }^{12,36}$ provocada por la proliferación fibrosa del ligamento amarillo, de origen aún incierto. Resnick $^{18,28,29,30}$ en la década de los 70 acuñó el término de "hiperostosis esquelética idiopática difusa" para referirse a la enfermedad de Forestier; fue el primero en llamar la atención en cuanto a la naturaleza sistémica del proceso ${ }^{42}$; definió criterios radiológicos específicos que todavía hoy se emplean:

1. Calcificación/osificación a lo largo de la región anterolateral de cuatro cuerpos vertebrales contiguos. 

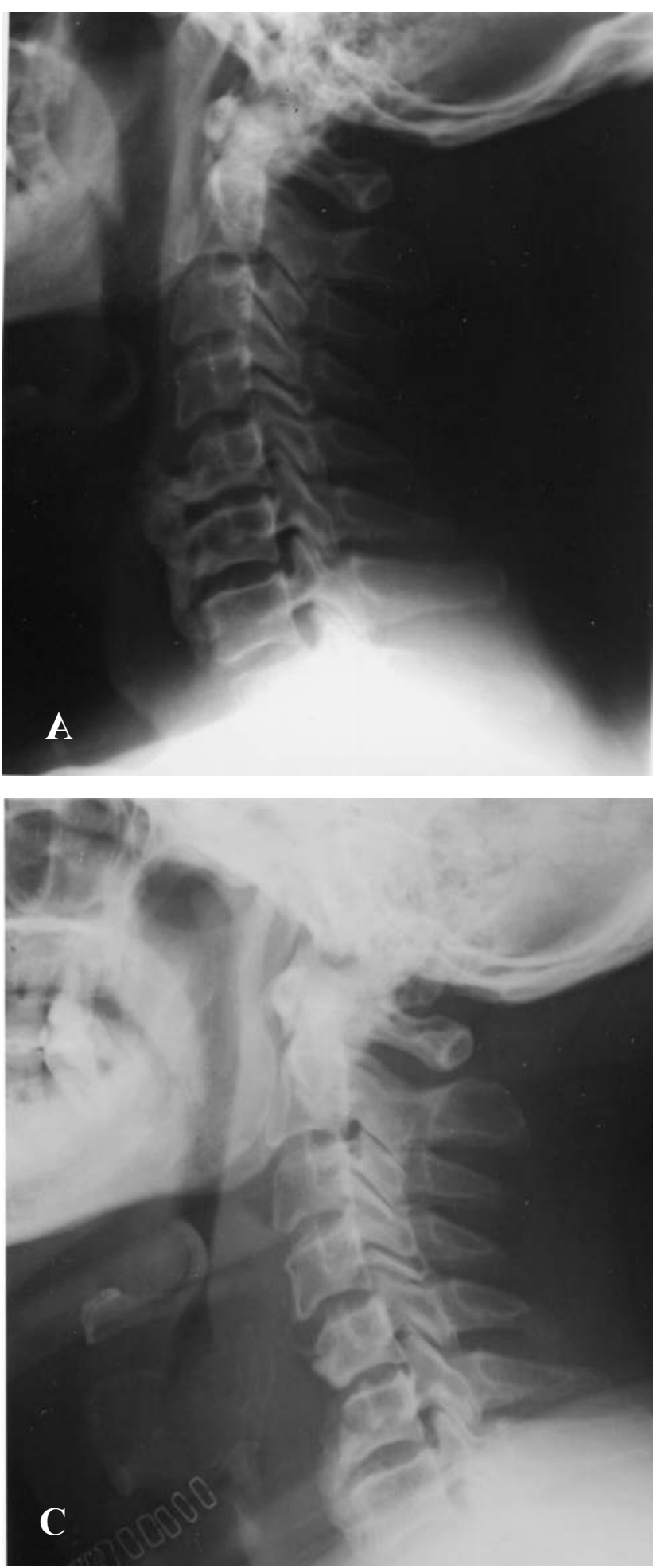

Figura 2 (Caso 2). A. Osificación del LLCA desde C5 hasta D1. B. Esofagograma que confirma un compromiso importante del tránsito a nivel de la osificación. C. Control postoperatorio mediante radiografia simple.
2008; 19: 350-355

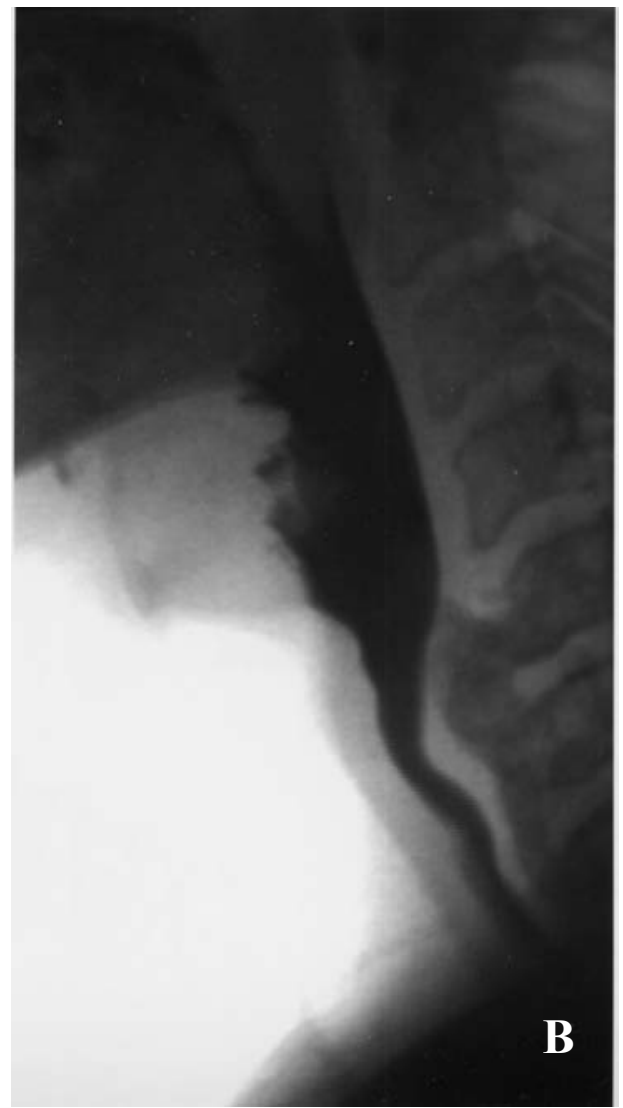

2. Respeto relativo de la altura del disco intervertebral en las áreas afectadas.

3. Ausencia de anquilosis articular apofisaria y esclerosis/fusión de la articulación sacroilíaca (criterios diagnósticos en la espondilitis anquilopoyética).

La enfermedad de Forestier no es un trastorno infrecuente en poblaciones de pacientes con enfermedades reumatológicas $^{30}$, pero es raro que éstos presenten síntomas que motiven la valoración por un neurocirujano. Aunque típicamente asintomáticos, existen casos documentados no sólo de problemas respiratorios, deglutorios o neurológicos, sino incluso de inestabilidad vertebral ${ }^{23}$. En la literatura neuroquirúrgica se encuentran pocos casos publicados, siendo la serie más larga la de McCafferty et al en $1995^{18}$, quienes describieron seis casos sintomáticos y un caso asintomático de esta enfermedad.

\section{Casos clínicos}

Caso 1. Paciente mujer de 84 años de edad que acude al Servicio de Urgencias por disnea, disfagia y odinofagia de 2 meses de evolución, con empeoramiento progresivo. Afebril. Valorada por ORL de guardia se diagnostica una epiglotitis aguda y la paciente ingresa para tratamiento. En la exploración fibroscópica describen epiglotis eritematosa y edematosa, cartílagos aritenoides edematosos, 
cuerdas vocales móviles, impronta de la pared posterior de la faringe sobre epiglotis con limitación para el paso aéreo, abundante mucosidad. Solicitan TC de cuello para descartar absceso retrofaríngeo que demuestra la existencia de un osteofito cervical anterior gigante a la altura del espacio intervertebral C3-C4, con efecto de masa importante sobre la faringe, junto con hiperostosis llamativa del LLCA. La exploración neurológica es normal, sin signos de mielopatía cervical. Ante los hallazgos la paciente es intervenida quirúrgicamente mediante un abordaje anterior convencional a la columna cervical; se realiza extirpación del osteofito descrito y fresado de la cara anterior vertebral de $\mathrm{C} 3$ y C4, sin complicaciones. No se objetivan adherencias entre el esófago y cuerpos vertebrales. La mejoría clínica de la paciente es inmediata tras la cirugía, con resolución completa de los síntomas hasta la actualidad (seguimiento 2 años).

Caso 2. Paciente varón de 54 años estudiado en Consultas Externas de Neurocirugía por cervicalgia de 2 años de evolución. En la radiografía simple y TC cervical se objetiva una proliferación ósea llamativa en cara anterior de cuerpos vertebrales de C5, C6, C7 y D1, en relación con la osificación del LLCA. Refiere alteraciones deglutorias en los últimos meses y sensación opresiva cervical con la flexión del cuello. El tránsito esófago-gastro-duodenal demuestra una impronta en la pared posterior del esófago cervical, con disminución del calibre esofágico. La exploración neurológica es normal. Se decide intervención quirúrgica mediante abordaje cervical anterior y se realiza la extirpación-fresado del osteofito descrito, sin complicaciones. Tras la intervención quirúrgica el paciente mejora de forma llamativa hasta la resolución completa de los síntomas (seguimiento 3 años y 2 meses).

\section{Discusión}

La enfermedad de Forestier o Hiperostosis esquelética idiopática difusa es una entidad relativamente común, según indican las tasas de prevalencia del 6 al 12\% publicadas en series de autopsia ${ }^{18,29}$. Afecta a hombres con más frecuencia que a mujeres $(2: 1)^{18,25}$, en grupos de edad media-avanzada; el pico de incidencia se da en la década de los 60 años de edad $6,7,28,29,32$. McCafferty et al publican en 1995 siete casos de enfermedad de Forestier, todos ellos varones mayores de 60 años ${ }^{18}$. Se trata de un trastorno sin preferencias geográfica, cultural ni genética ${ }^{35}$.

Es una enfermedad de etiología desconocida ${ }^{7,9,13,29}$; en la década de los 50 se expuso la inmovilización vertebral como causa de la hiperostosis extensa ${ }^{22,34}$; una diátesis osificante también se consideró un factor etiológico. En algunas publicaciones se sugiere que la osificación ligamentaria ocurre en un intento de conseguir rigidez. Suzuki et al demuestran en $1991^{39}$ que la calcificación del LLCA continúa hasta la eliminación del movimiento en los segmentos adyacentes. Se han publicado varios casos de osificación recurrente después de la exéresis de osteofitos ${ }^{3,38,41}$; sin embargo, la región torácica es el segmento más inmóvil de la columna y la más frecuentemente afectada. De igual modo, Oga et al explican que la movilidad cervical incrementada puede contribuir a la progresión de la osificación ${ }^{21}$. Suzuki ${ }^{39}$ presenta 11 pacientes con hiperostosis esquelética idiopática difusa y encuentra que el movimiento cervical se correlaciona directamente con la progresión más rápida de la osificación; explica la necesidad de inmovilizar los segmentos afectados, tras la extirpación de la osificación intervertebral, para prevenir su recurrencia. La mayoría de pacientes permanecen asintomáticos; dolor articular leve y dolor/rigidez de espalda son, con frecuencia, los únicos síntomas ${ }^{6,18,29}$, poco importantes en la población anciana que a menudo sufre otras enfermedades reumatológicas o enfermedad degenerativa de la columna ${ }^{18}$. Cuando está implicada la columna cervical, la disfagia es el síntoma de presentación más común, descrita por primera vez por Mosher en $1926^{20}$; rara vez la disfagia está causada por osteofitos anteriores en la columna dorsal ${ }^{38,39,40}$.

Aproximadamente el $30 \%$ de pacientes refieren dificultad para la deglución ${ }^{15}$ y el $10 \%$ requieren intervención quirúrgica, ${ }^{5,8,10,18,30}$. Es inusual la presencia de síntomas neurológicos; sin embargo, se ha descrito compromiso de raíces nerviosas y médula espinal ${ }^{1,17,31,43}$, atrapamiento de nervios periféricos ${ }^{36}$, síndrome del desfiladero torácico ${ }^{36}$, síndrome de Horner $^{32}$, parálisis del nervio recurrente laríngeo ${ }^{3,14}$ e insuficiencia arterial vertebral atribuidos a la misma. En 1995 Goto et $\mathrm{al}^{11}$ presentan un caso de enfermedad de Forestier que causaba mielopatía cervical y tetraparesia secundaria; una pseudoartrosis entre el tubérculo posterior del atlas y la apófisis espinosa del axis provocaba la compresión extradural de la médula cervical. Patel publica en el $2002^{25}$ cinco casos de estenosis espinal severa en la unión craneocervical; en todos ellos objetivó la osificación del LLCA entre C3 y C7 y demostró la existencia de una masa de tejido blando retroodontoidea; en cuatro pacientes se realizó odontoidectomía transoral y extirpación de la masa hipertrófica y en un paciente se realizó una descompresión posterior; los autores postulan que el desarrollo de un pannus hipertrófico retroodontoideo en estos pacientes se debe a una biomecánica alterada en la unión atlantoaxial ${ }^{24}$.

El tamaño de los osteofitos no se correlaciona con la severidad de los síntomas, según expone $\mathrm{McCafferty}{ }^{18}$. Es importante destacar que la disfagia causada por osteofitos cervicales anteriores es una entidad infrecuente, siendo la disfagia de origen maligno mucho más común.

En cuanto al tratamiento adecuado de la disfagia existe controversia en los diferentes trabajos publicados. Algunos autores proponen observación, modificación de la dieta y 
medicación antiinflamatoria ${ }^{2,12,17,26,31,32}$, quizás una actitud prudente en presencia de síntomas leves; en casos graves debe realizarse la descompresión quirúrgica anterior. En algunos artículos publicados se propone la necesidad de una fusión cervical anterior, además de la extirpación de los osteofitos existentes; ello deriva de la hipótesis publicada por Suzuki et $\mathrm{al}^{39}$ acerca de que la osificación de ligamentos progresa hasta el grado de rigidez; de esa forma, la recurrencia sería probable si no se realiza una fusión cervical. Hirano presentó en $1982^{13}$ el caso de un paciente que desarrolló un osteofito recurrente en la región intervenida ocho años y medio antes; insiste en la necesidad de realizar un seguimiento de los pacientes intervenidos a largo plazo. Igualmente Suzuki ${ }^{39}$ publicó que uno de sus dos pacientes necesitó reintervención quirúrgica once años después de la cirugía inicial ante la recurrencia de la osificación del LLCA y disfagia secundaria. No obstante, la recurrencia después de 8-11 años no debe preocuparnos en exceso en relación con una población anciana y su esperanza de vida. La morbilidad asociada con el procedimiento quirúrgico es mínima y todos los pacientes refieren mejoría significativa de su calidad de vida.

La compresión o distorsión mecánica del esófago provoca disfagia ${ }^{16}$; sin embargo, si ésta fuera la única causa de la disfagia en la enfermedad de Forestier, la extirpación osteofitaria conseguiría un alivio inmediato de los síntomas en todos los pacientes. Ello sugiere que otros factores están implicados en la disfunción de los mecanismos de la deglución $^{4,18,19,33,39}$; estos pueden ser cambios inflamatorios crónicos asociados con fibrosis, tumores o traumatismos ${ }^{15,19,37}$. La compresión del esófago por osteofitos produce inflamación en la pared, que podría originar dificultad en la deglución ${ }^{2,3,12,17,21,26,38,43}$; la acumulación de tejido fibroso en la pared del esófago, secundaria a una inflamación crónica, comprometería la peristalsis esofágica. Además, se ha postulado que la compresión del esófago a largo plazo y la inflamación secundaria provocarían una disrupción del plexo nervioso esofágico ${ }^{38}$. Por ello, es posible que compresión mecánica e inflamación crónica contribuyan a la producción de disfagia. La resolución inmediata de los síntomas se debe a la eliminación del obstáculo mecánico mediante la exéresis osteofitaria, mientras que la mejoría lenta que experimentan otros pacientes se debe a la inflamación/fibrosis en la pared esofágica debida a la compresión a largo plazo. Esto último puede hacernos pensar que el tratamiento quirúrgico en una fase temprana quizás sea la opción más adecuada.

\section{Bibliografía}

1. Alenghat, J.P., Hallet, M., Kido, D.K.: Spinal cord compression in diffuse idiopathic skeletal hyperostosis. Radiology 1982; 142: 119-120.
2. Bauer, F.: Dysphagia due to cervical espondylosis. J Laryngol Otol 1953; 67: 615-630.

3. Bulos, S.: Dysphagia caused by cervical ostephyte. J Bone Joint Surg 1974; 56B: 148-152.

4. Epstein, N.E., Hollingsworth, R.: Ossification of the cervical anterior longitudinal ligament contributing to dysphagia. J Neurosurg (Spine 2) 1999; 90: 261-263.

5. Eviatar, E., Harell, M.: Diffuse idiopathic skeletal hyperostosis with dysphagia (A review). J Laryngol Otol 1987; 101: 627-632.

6. Forestier, J., Rotes-Querol, J.: Senile ankylosing hyperostosis of the spine. Ann Rheum Dis 1950; 9: 321-330.

7. Forestier, J., Lagier, R.: Ankylosing hyperostosis of the spine. Clin Orthop 1971; 74: 65-85.

8. Gamache, F.W., Voorhies, R.M.: Hypertrophic cervical osteophytes causing dysphagia. A review. J Neurosurg 1980; 53: 338-344.

9. Gaucher, A.A., Péré, P.G., Gillet, P.M.: From ankylosing spondylitis to Forestier's disease: ossifying enthesopathy, a unifying concept. J Rheumatol 1990; 17: 854-856.

10. Goffin, J., Van Calenbergh, F.: Forestier's disease. J Neurosurg 1996; 85: 524-525.

11. Goto, S., Tanno, T., Moriya, H.: Cervical myelopathy caused by pseudoarthrosis between the atlas and axis associated with diffuse idiopathic skeletal hyperostosis. Spine 1995; 20: 2572-2575.

12. Hargrove, M.D., Jr.: Dysphagia associated with inflammatory reaction within the esophagus at the level of a vertebral spur. Gastrointes Endosc 1966; 28-29.

13. Hirano, H., Suzuki, H., Sakakibara, T., Higuchi, Y., Inoue, K., Suzuki, Y.: Dysphagia due to hypertrophic cervical osteophytes. Clin Orthop 1982; 167: 168-172.

14. Karlins, N.L., Yagan, R.: Dyspnea and hoarseness. A complication of diffuse idiophatic skeletal hyperostosis. Spine 1991; 16: 235-237.

15. Kissel, P., Youmans, J.R.: Posttraumatic anterior cervical osteophyte and dysphagia: surgical report and literature review. J Spinal Disord 1992; 5: 104-107.

16. Kritzer, R.O., Parker, W.D.: DISH: A cause of anterior cervical ostephyte-induced dysphagia. Spine 1988; 13: 130132.

17. Lambert, J.R., Tepperman, P.S., Jimenez, J.: Cervical spine disease and dysphagia. Am J Gastroenterol 1981; 76: 3540.

18. McCafferty, R.R., Harrison, M.J., Tamas, L.B., Larkins, M.V.: Ossification of the anterior longitudinal ligament and Forestier's disease: an analysis of seven cases. J Neurosurg 1995; 83: 13-17.

19. McGarrah, P.D., Teller, D.: Posttraumatic cervical osteophytosis causing progressive dysphagia. South Med J 1997; 90: 858-860.

20. Mosher, H.P.: Exostosis of the cervical vertebrae as a cause for difficulty in swallowing. Laryngoscope 1926; 36: 
181.

21. Oga, M., Mashima, T., Iwakuma, T., Sugioka, Y.: Dysphagia complications in ankylosing spinal hyperostosis and ossification of the posterior longitudinal ligament. Spine 1993; 18: 391-394.

22. Oppenheimer, A.: Calcification and ossification of vertebral ligaments (spondylitis ossificans ligamentosa); roentgen study of pathogenesis and clinical significance. Radiology 1942; 38: 160-173.

23. Paley, D., Schwartz, M., Cooper, P., Harrins, W.R., Levine, A.M.: Fractures of the spine in diffuse idiopathic skeletal hyperostosis. Clin Orthop 1991; 267: 22-32.

24. Panjabi, M.M., Vasavada, A., White A.A.: Cervical spine biomechanics. Semin Spine Surg 1993; 5: 10-16.

25. Patel, N.P., Wright, N.M., Choi, W.W., McBride, D.Q., Johnson, P.: Forestier disease associated with a retroodontoid mass causing cervicomedullary compression. J Neurosurg (Spine 2) 2002; 96: 190-196.

26. Ratnesar, P.: Dysphagia due to cervical exostosis. Laryngoscope 1970; 80: 469-471.

27. Reisner, A., Stiles, R.G., Tindall, S.C.: Diffuse idiopathic skeletal hyperostosis causing acute thoracic myelopathy: a case report and discussion. Neurosurgery 1990; 26: 507511.

28. Resnick, D., Shaul, S.R., Robins, J.M.: Diffuse idiopathic skeletal hyperostosis (DISH): Forestier's disease with extraspinal manifestations. Radiology 1975; 115: 513-524.

29. Resnick, D., Niwayama, G.: Radiographic and pathologic features of spinal involvement in diffuse idiopathic skeletal hyperostosis (DISH). Radiology 1976; 119: 559-568.

30. Resnick, D., Shapiro, R.F., Wiesner, K.B., Niwayama, G., Utsinger, P.D., Shaul, S.R.: Diffuse idiopathic skeletal hyperostosis (DISH) (Ankylosing hyperostosis of Forestier and Rotes-Querol). Semin Arthritis Rheum 1978; 7: 153-187.

31. Saffouri, M.H., Ward, P.H.: Surgical correction of dysphagia due to cervical osteophytes. Ann Otol Rhinol Laryngol 1974; 83: 65-70.

32. Saunders, W.H.: Cervical osteophytes and dysphagia. Ann Otol Rhinol Laryngol 1970; 79: 1091-1097.

33. Shaw, C.B., Bawa, R., Snider, G., Wax, M.K.: Traumatic retropharyngeal hematoma: a case report. Otolaryngol
Head Neck Surg 1995; 113: 485-488.

34. Smith, C.F., Pugh, D.G., Polley, H.F.: Physiologic vertebral ligamentous calcification. An aging process. Am J Roentgenol 1955; 74: 1049-1058.

35. Spagnola, A.M., Bennett, P.H., Terasaki, P.I.: Vertebral ankylosing hyperostosis (Forestier's disease) and HLA antigens in PIMA Indians. Arthritis Rheum 1978; 21: 467-472.

36. Stechison, M.T., Tator, Ch.H. : Cervical myelopathy in diffuse idiopathic skeletal hyperostosis. Case report. J Neurosurg 1990; 73: 279-282.

37. Stewart, M., Johnston, R.A., Stewart, I., Wilson, J.A.: Swallowing performance following anterior cervical spine surgery. Br J Neurosurg 1995; 9: 605-609.

38. Stuart, D.: Dysphagia due to cervical osteophytes. A description of five patients and a review of the literature. Int Orthop 1989, 13: 95-99.

39. Suzuki, K., Ishida, Y., Ohmori, K.: Long term followup of diffuse idiopathic skeletal hyperostosis in the cervical spine. Analysis of progression of ossification. Neuroradiology 1991; 33: 427-431.

40. Underberg-Davis, S., Levine, M.S.: Giant thoracic osteophyte causing esophageal food impaction. Case report. AJR 1991; 157: 319-320.

41. Warnick, C., Sherman, M.S., Lesser, R.W.: Aspiration pneumonia due to diffuse cervical hyperostosis. Chest 1990; 98: 763-764.

42. Weisz, G.M.: Ossifying surgical scar in Forestier's disease. Int Surg 1985; 70: 273.

43. Zerhouni, E.A., Bosma, J.F., Donner, M.W. : Relationship of cervical spine disorders to dysphagia. Dysphagia 1987; 1: 129-144.

Alcázar, J.; Jerez, P.; Gómez-Angulo, J.C.; Tamarit, M.; Navarro, R,; Ortega, J.M.; Aragonés, P.; Salazar, F.; del Pozo, J.M.: Enfermedad de Forestier-Rotes-Querol. Osificación del ligamento longitudinal cervical anterior como causa de disfagia. Neurocirugía 2008; 19: 350-355 .

Correspondencia postal: Lucía Alcázar Vaquerizo. Servicio de Neurocirugía. Hospital Universitario de Getafe. Ctra. Toledo s/n, km. 12.500. 28905 Getafe. Madrid. 